\title{
Enhancing visuospatial performance through video game training to increase learning in visuospatial science domains
}

\author{
Christopher A. Sanchez
}

Published online: 25 October 2011

(C) Psychonomic Society, Inc. 2011

\begin{abstract}
Although previous research has demonstrated that performance on visuospatial assessments can be enhanced through relevant experience, an unaddressed question is whether such experience also produces a similar increase in target domains (such as science learning) where visuospatial abilities are directly relevant for performance. In the present study, participants completed either spatial or nonspatial training via interaction with video games and were then asked to read and learn about the geologic topic of plate tectonics. Results replicate the benefit of playing appropriate video games in enhancing visuospatial performance and demonstrate that this facilitation also manifests itself in learning science topics that are visuospatial in nature. This novel result suggests that visuospatial training not only can impact performance on measures of spatial functioning, but also can affect performance in content areas in which these abilities are utilized.
\end{abstract}

Keywords Cognitive training - Visuospatial abilities .

Science learning

A long tradition of factor-analytic research has provided strong evidence that there exists a set of visuospatial abilities that can be differentiated from other cognitive functions, such as verbal ability or attention, and represent a complex of multiple, distinguishable, spatial faculties (see Hegarty \& Waller, 2006, for a review). Classically, investigations into these visuospatial abilities identify two primary, but highly related, spatial capacities

C. A. Sanchez $(\square)$

Cognitive Science and Engineering, Arizona State University,

7001 E. Sonoran Arroyo Mall,

Mesa, AZ 85202, USA

e-mail: c.sanchez@asu.edu within normal human populations (Carroll, 1993; Just \& Carpenter, 1985; Stumpf \& Eliot, 1995).

The first (and usually strongest) visuospatial marker represents the ability to transform or manipulate existing relationships into new wholes, commonly referred to as spatial visualization (Mumaw, Pellegrino, Kail, \& Carter, 1984; Pellegrino \& Hunt, 1991). Spatial visualization performance is typified by such measurement tasks as the paper folding and form board measures (French, Ekstrom, \& Price, 1963), in which the individual must not only preserve existing spatial relationships, but also manipulate said spatial relationships into a revised new representation. The second primary spatial facet represents the ability of individuals to preserve spatial relations and orientations, independently of egocentric viewpoint (Cooper, 1975; Cooper \& Shepard, 1973). This aptitude is often captured by cube and figure rotation tasks, where the individual must mentally rotate the spatial representation, while preserving the spatial relations within the item. Recent research has also identified other additional discrete spatial abilities related to wayfinding, egocentric viewpoints, or perspective taking that are likewise used to engage in spatial reasoning with the outside world (Hegarty \& Waller, 2006; Kozhevnikov \& Hegarty, 2001; Zacks, Mires, Tversky, \& Hazeltine, 2002). These spatial abilities have been connected to performance in other complex spatial domains, such as using maps (Sanchez \& Branaghan, 2009), air-traffic control (Ackerman, 1992), and even comprehension of narrative texts where readers follow the actions of a character in physical space (Bower \& Morrow, 1990; Fincher-Kiefer, 2001; Fincher-Kiefer \& D'Agostino, 2004; Haenggi, Kintsch, \& Gernsbacher, 1995).

Interestingly, it has been suggested that visuospatial aptitudes can be enhanced via dedicated training. For 
example, playing video games like "Tetris," or first-person shooter (FPS) titles has produced stable performance gains on visuospatial ability tests (Cherney, 2008; Feng, Spence, \& Pratt, 2007; Okagaki \& Frensch, 1994; Terlecki, Newcombe, \& Little, 2008). Similar effects have also been observed for other training regiments that include activities such as organized athletics (Ginn \& Pickens, 2005), dental education (Hegarty, Keehner, Khooshabeh, \& Montello, 2009), or even practice on spatial tasks themselves (Wright, Thompson, Ganis, Newcombe, \& Kosslyn, 2008). While these tasks differ significantly in their goals, physical requirements, and structure, importantly, these tasks all share the same basic requirement: to manipulate and use visuospatial information repeatedly in a goal-directed way. This experience likely improves spatial function (Feng et al., 2007; Okagaki \& Frensch, 1994), which produces the observed increase in visuospatial test scores.

However, while these training studies have shown that performance on simple visuospatial tests does improve with training, a critical issue relative to the evaluation of the effectiveness of this training has been overlooked: Namely, does this spatial training also enhance performance in other domains in which visuospatial processing is relevant for performance? To date, no study has explored the link between potential enhancement of these spatial processes and increases in performance in other relevant target areas.

This is to say not that video game training (broadly construed) has not led to observed enhancements in performance in other visuospatial tasks but, simply, that the connection with changes in visuospatial performance (measured through assessments) has not been explicitly made to these increases. For example, video games and simulations have been shown to enhance real-world performance in terms of manned flight (Gopher, Weil, \& Bareket, 1994), golf-putting (Fery \& Ponserre, 2001), and reaction time to visual stimuli (Dye, Green, \& Bavelier, 2009; Green \& Bavelier, 2003). Furthermore, video game experience has been correlated with better performance in laproscopic surgery tasks (Rosenberg, Landsittel, \& Averch, 2005; Rosser et al., 2007). The present study seeks to build on these results and connect improvements in complex performance realized through video game experience to measureable improvements in visuospatial processing.

\section{Visuospatial abilities and science learning}

Recent research has suggested a link between learners' inherent spatial aptitudes and their ability to succeed in learning physical science concepts. Learners who are more adept at mentally manipulating this spatial information have been shown to better learn earth science (Black, 2005; Sanchez \& Wiley, 2010; Sibley, 2005), chemistry (Bodner \& McMillen, 1986; Carter, LaRussa, \& Bodner, 1987; Pribyl \& Bodner, 1987; Wu \& Shah, 2004), and math (Friedman, 1995) and to better understand physical systems (Hegarty \& Sims, 1994; Hegarty \& Steinhoff, 1997; Kozhevnikov, Motes, \& Hegarty, 2007). Since these STEM disciplines involve learning about actual physical, spatial phenomena, it is perhaps not surprising that the ability to internalize and manipulate visuospatial information is related to success in these domains (Kozma \& Russell, 2005; Mathewson, 1999) and the creation of spatial mental models of understanding for these content areas (Tversky, 1991).

For example, in order for learners to successfully understand the geological phenomena of plate tectonics, they must first identify the relevant conceptual units that are spatial in nature (e.g., plates, magma, plate boundaries) and then also identify how these units interact and are related to one another. Ultimately, the learner must integrate and represent these discrete spatial units within their own internal, runnable, mental model that should behave in a consistent fashion with the actual material being learned (Gentner \& Stevens, 1983; Hegarty, 1992). Indeed, spatial faculties have been found to be relevant for the creation of these complex spatial situation models and the formation of other abstract spatial representations (Friedman \& Miyake, 2000; Haenggi et al., 1995). Obviously, the more accurately the learner can mentally construct the spatial situation or phenomena (relative to the actual phenomenon), the better the learner likely understands the given material.

Importantly, if these spatial faculties are critical for the acquisition and subsequent development of appropriate spatial mental models, improving the efficiency of these spatial processes through training should likewise enhance the quality of learning and, similarly, the likelihood to continue to pursue further instruction in these STEM fields. In other words, if one accepts that visuospatial faculties are critical for the development of understanding in physical science, does improving the efficiency of visuospatial processes through training also enhance the quality of learning in STEM fields? Thus, science learning provides an appropriate criterion for exploring whether spatial performance gains realized through training likewise increase performance in a target domain.

In the present study, different visuospatial training groups were compared as they learned about the science topic of plate tectonics. Participants' visuospatial ability was assessed before and after training, and working memory capacity was also assessed to control for differences in general cognitive ability (Conway et al., 2005). If spatial training does, in fact, increase the efficiency of 
visuospatial processing, performance increases in both visuospatial processing and science learning should be realized for the spatial-training group, relative to a nonspatial-training control. However, if these spatial-training results do not provide a global benefit for visuospatial processing in all relevant areas, one would expect to see increases in performance on spatial tests, but not enhancement in more general content areas (e.g., science learning).

\section{Method}

Participants

Sixty native English speakers from a large public university were solicited for participation and were compensated with class credit in an introductory psychology course.

\section{Materials}

Prior domain knowledge To evaluate the initial level of prior knowledge of plate tectonics, all participants completed a Volcano Concept Pretest (VCP) that has been used previously to evaluate prior understanding of plate tectonics (Wiley et al., 2009). The VCP consists of 30 inferences relevant to the domain area, half of which are true and the remaining half of which are false. Participants were asked to correctly identify each of the inference items as true or false and were awarded a single point for every correct identification. Higher scores on the VCP are indicative of higher knowledge in the content area.

Visuospatial ability pretest All participants completed Part I of two measures of visuospatial ability: the Paper Folding (VZ-2) and Card Rotations (S-1; French et al., 1963) Tests. Part I of the VZ-2 task consists of 10 trials on which the participants are shown a diagram of an irregularly folded piece of paper, with all requisite folds marked. The participants are told to imagine a single hole being punched through all the layers of paper at an indicated point and are then are asked to choose between five possible responses as to what the paper would look like when completely unfolded. Participants have $3 \mathrm{~min}$ to complete this initial set. The final score on this test is the number of correct items (maximum score of 10), and this is considered a spatial visualization task.

Part I of the S-1 task consists of a set of 10 trials on which participants are asked to judge whether each of the eight possible options represent either a rotation or a mirror image of a given shape. Participants are awarded a single point for every correct judgment across the 10 trials (maximum score of 80 ). This initial set also takes $3 \mathrm{~min}$, and this task is considered a measure of spatial relations.

Working memory capacity To control for differences in general cognitive ability, all participants completed a computerized assessment of working memory capacity (WMC): Automated Operation Span (AOSpan; Unsworth, Heitz, Schrock, \& Engle, 2005). In the AOSpan task, participants are shown a simple equation followed by a letter [e.g., IS (8/4) $1=1$ ? C] and are asked to evaluate the correctness of the equation while remembering the letter for a later test. Equation-letter strings were presented in sets that contained from two to seven strings. At the end of each set, participants were prompted to identify, in the correct serial order, those letters that followed the equations and were awarded a single point for every letter recalled in the correct serial position. Participants completed three trials of each set size, and the order of these sets was randomized. All administration and scoring followed the recommendations of Unsworth et al.

Training and game experience survey Participants were randomly assigned to either the spatial-training group ( $n=30 ; 40 \%$ female), which played the FPS Halo: Combat Evolved (Microsoft, 2001), or instead the nonspatial-training condition $(n=30 ; 36.7 \%$ female), which played the game Word Whomp'(Electronic Arts, 2011), where participants instead make words out of a group of six random letters to earn points. These games were played on desktop PCs, with 19-in. monitors. Screenshots are available in Fig. 1. As described above, FPS video games have been used previously to train visuospatial performance (Feng et al., 2007). After training, participants then rated their training experience for "fun" on a 1-10 scale (1 being lowest) and also reported how many hours a week, on average, they normally played video games.

Text, essay measure, and visuospatial posttest Participants read a complex text about plate tectonics that contained $\sim 3,500$ words and no illustrations (Wiley et al., 2009). The text was based on information from the USGS "This Dynamic Planet" unit and the NASA Classrooms of the Future "Volcanoes" unit, originally developed by Wiley (2001). It included five major concepts from a causal model of volcanic eruptions: (1) Heat currents cause plates to move and interact; (2) oceanic plates subduct beneath continental plates; (3) continental plates melt into viscous gaseous magma; (4) more buoyant than surrounding magma, new magma fills magma chambers; (5) pressure builds in magma chambers and is released (Sanchez \& Wiley, 2010). 
Fig. 1 Screenshots of the video games used in either training condition. The screenshot of the non-spatial-training condition appears at the top (Word Whomp $\odot$, EA Games), and the screenshot for the spatial-training condition (Halo: Combat Evolved $\subset$, Microsoft) appears at the bottom
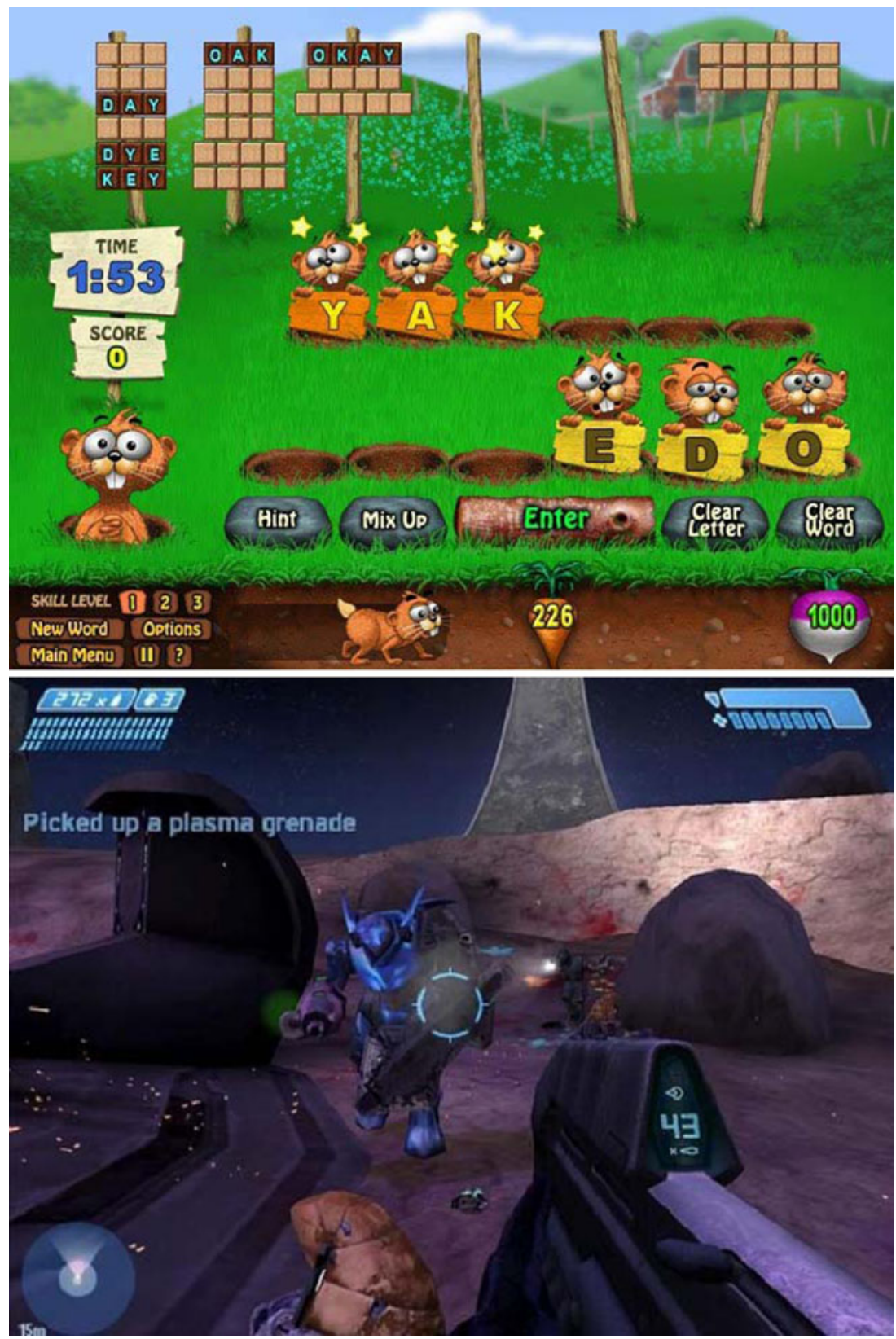

After reading, participants wrote a causal essay about "What caused Mt. St. Helens to erupt?" that was then evaluated for the presence of the five a priori causal concepts. Since the text does not explicitly mention the circumstances of Mt. St. Helens, participants are required to identify and apply the relevant concepts from the text to this novel instance. Essays were evaluated for the presence of these five a priori concepts and were given 1 point for every correct concept included in the response, with a maximum score on the essay measure being 5 . Two independent scorers blind to experimental condition coded these essay responses for the presence of these concepts and produced a high level of interrater agreement $(\mathrm{ICC}=.91, p<.01)$. Any scoring differences were resolved through discussion.
Participants also completed a spatial posttest, which consisted of Part II of the VZ-2 and S-1 tasks. These measures are identical in format and administration to those sets used to assess initial visuospatial abilities, except that they included completely novel items. This second administration was designed to evaluate any change in performance on visuospatial measures.

\section{Procedure}

Participants first completed the VCP and then completed Part I of both the VZ-2 and S-1 tasks (randomized). Based on condition, participants then engaged in $25 \mathrm{~min}$ of training, after which they rated their gaming experience. 
Participants then read the target science text for $17 \mathrm{~min}$ and then completed the essay task. Importantly, the website was unavailable to the participants while they were completing the essay task. Participants then completed Part II of both visuospatial tasks (randomized). The entire experiment took no longer than $1.5 \mathrm{~h}$. WMC was evaluated in a separate half-hour session.

\section{Results and discussion}

Descriptive statistics for all measures, by training group, are available in Table 1. As is visible in Table 1, there were no reliable differences $(t \mathrm{~s}<1.10)$ between the spatial-training groups on any of the pretesting measures, including prior knowledge, WMC, and initial visuospatial ability as measured by Part I of either the VZ-2 or the S-1 task. These findings suggest that the different training groups were well matched on ability and content knowledge. Furthermore, both groups also did not significantly differ $(t \mathrm{~s}<1)$ in either their rating of how "fun" they thought the training was or the average number of hours they played video games each week. This suggests that any subsequent results are not due to motivation or other video-gamerelated familiarity factors.

In terms of visuospatial performance, there were no reliable main effects or interactions for the VZ-2 task ( $p$ s $>.05)$. However, a mixed ANOVA on the S-1 task indicated that while there was no overall difference between training groups, $F(1,58)=1.76, p>.05$, there was a significant increase in performance on the S-1 task prepost, $F(1,58)=13.30, p<.01, \eta_{\mathrm{p}}{ }^{2}=.19$. There was also a significant interaction between group and time of testing, $F(1,58)=4.32, p<.05, \eta_{\mathrm{p}}{ }^{2}=.07$ (see Fig. 2a). Planned comparisons indicate that while there was no difference pre-post for the non-spatial-training group, $t<1.31$, there was a significant improvement for the spatial-training group, $t(29)=3.76, p<.01$. This suggests that the prepost main effect was likely driven by performance
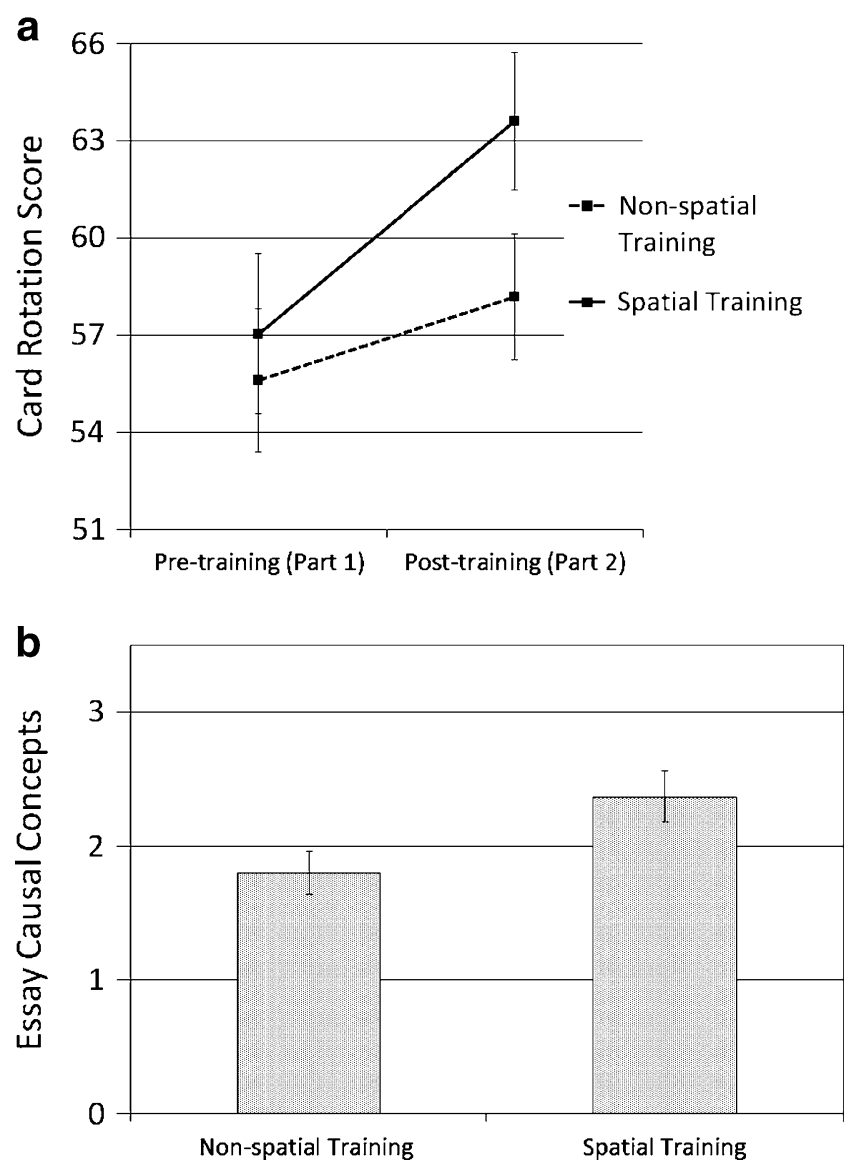

Fig. 2 a Performance on the visuospatial ability measure before and after training for each group. b Difference in performance on the essay task for each group. Error bars represent the standard errors of the means

increases in the training group. This finding is consistent with prior research that has shown a benefit for playing FPS and enhancing performance on speeded rotation measures (Feng et al., 2007). One should also not be surprised that there was no training effect in the VZ-2 task, since the primary spatial experience in FPS involves only constant mental rotation in three dimensions and does not
Table 1 Mean $(S D)$ performance for each variable by training group

\begin{tabular}{lcc}
\hline Measure & Non-Spatial Training & Spatial Training \\
\hline WMC & $57.20(9.22)$ & $56.13(13.47)$ \\
Prior knowledge & $19.77(2.76)$ & $20.37(3.01)$ \\
VZ-2 (Part I) & $5.47(2.18)$ & $6.03(1.81)$ \\
VZ-2 (Part II) & $6.10(1.97)$ & $5.87(1.85)$ \\
S-1 (Part I) & $55.60(13.55)$ & $58.17(12.12)$ \\
S-1 (Part II) & $57.03(11.55)$ & $63.60(10.60)$ \\
Essay task & $1.80(0.89)$ & $2.37(1.07)$ \\
How "fun" was training? & $6.90(2.23)$ & $7.00(2.00)$ \\
How many hours per week do you play video games? & $4.68(6.74)$ & $4.70(5.33)$ \\
\hline
\end{tabular}


contain any requirement that the player visualize or transform spatial information. This divergence in training effect, relative to task requirements, is consistent with prior research that has suggested that only those spatial faculties that are explicitly targeted by training will show improvement (Okagaki \& Frensch, 1994).

In terms of learning science concepts, a simple ANOVA indicated that participants in the spatial-training group also significantly outperformed the non-spatial-training group on the essay task, $F(1,58)=4.31, p<.05, \eta_{\mathrm{p}}{ }^{2}=.07$ (see Fig. 2b). This result, when considered in tandem with the improvement in visuospatial scores and the lack of a difference in prior knowledge of the content area on the pretest, suggests that the improvement of visuospatial performance realized through the training manipulation also enhanced the likelihood of learning science concepts.

Overall, these results replicate the previously found facilitation of spatial training on visuospatial test performance-specifically, spatial relations (Feng et al., 2007). However, they extend these findings and suggest that training that enhances visuospatial processing can likewise improve performance in a related target domain like science. This suggests a general benefit for such training, which is not isolated to visuospatial task performance alone.

However, it is worth considering alternative explanations of the improvement realized in the training group. First, could these results simply be due to different levels of engagement or effort produced by the different training conditions? While possible, this explanation seems unlikely, since participants rated both games as equally "fun." Since the games themselves are likewise equally unrelated in content and task goals to the target science-learning task, one would be hard pressed to argue that the interest in one of the games produced a fundamentally different engagement with the science text than did the other.

One might also consider that the training manipulation simply "warmed up" or activated dormant spatial processes or ways of thinking and, thus, did not produce a traditional training benefit. While possible given the somewhat brief training manipulation used here, this explanation seems unlikely for a few reasons. First, while this alternative explanation might certainly account for higher performance on the target essay task, it is unclear how it would also account for the improvement in performance on the spatial measure-specifically, the S-1 task. The visuospatial assessments used here have been implemented numerous times across their nearly 50-year existence and have been consistently recognized as accurate indicators of an individual's visuospatial ability. One critical characteristic that has enabled such recognition is their high reliability across their constituent Parts I and II, which again consist of different but conceptually similar items across discrete sets
(Cronbach's $\alpha>\sim .80$; for recent examples that include both the S-1 and VZ-2 tasks, see Kozhevnikov \& Hegarty, 2001; Kozhevnikov et al., 2007; Miyake, Friedman, Rettinger, Shah, \& Hegarty, 2001). If there was any possibility that unutilized visuospatial faculties somehow could be primed or activated at will (i.e., between parts, for example), this reliability across parts would be much reduced and would likely have manifested itself at some point in their long history, which to date it has not. After all, if one accepts this activation explanation for the improvement in visuospatial scores, the most obvious, and powerful, prime or suggestion for activating spatial processes would, of course, be the first part of the visuospatial tests themselves. Given that this direct prime has so far failed to produce an enhancement on previous administrations of these visuospatial assessments (and likewise, here, in the nontraining condition), it would be difficult to explain how more diffuse primes (e.g., FPS video game experience) could accomplish this. Thus, the training effect found here is unlikely to be a result of such a prime or activation, since it cannot explain improvement in both the essay and visuospatial tasks. The more likely explanation is that there has been some kind of change or enhancement of visuospatial thinking (specifically, spatial relations in this experiment), which subsequently changes how individuals process visuospatial information. This cognitive change must then be what produces the essay effect and, likewise, improves visuospatial test performance. Certainly, one piece of evidence that would further affirm this cognitive change explanation would be a demonstration of a durable and lasting change in visuospatial performance over time. While such an investigation is beyond the scope of this study, it offers a promising avenue for future investigation. For example, it would be interesting to see whether the training effect observed here can be optimized to produce even greater magnitudes of performance change that persist over a substantial period of time, and at what point additional training time fails to increase said gains and, instead, begins to produce diminishing returns. Similarly, transfer of training to other relevant visuospatial tasks would also serve as strong evidence for this change explanation.

Finally, it is also worth mentioning that the present study extends work in the visuospatial-training literature in that it produced a verifiable and theoretically consistent effect across two tasks (e.g., S-1 and essay), utilizing only a small window of visuospatial training. While most studies on this topic implement hours of training, it must be mentioned that, to date, no study has empirically investigated the duration of spatial training and whether such long (or short) durations are better or worse for producing changes in visuospatial performance. The present finding represents a step in this direction by suggesting that smaller training windows do also have some utility, and future research 
might further explore the boundaries of these training durations and their ultimate effectiveness.

In terms of relevant applications, these results suggest that appropriate experience can be used to augment the learning scenario and effectively increase understanding in a critical target domain. Furthermore, this also suggests that perhaps such training could be used to attenuate individual differences in relevant cognitive abilities-specifically, for those individuals who might most benefit from an increase in visuospatial processing.

Acknowledgments The author would like to thank Carrie R. Norris and Jennifer Wiley for their initial thoughts on the overall project and would also like to thank Pedro Gutierrez, Shaun Lobsinger, and Jason Mamangon for their help with data collection.

This research was supported in part by an Arizona State University CTI Scholarship Support and Enhancement Grant to the author.

\section{References}

Ackerman, P. L. (1992). Predicting individual differences in complex skill acquisition: Dynamics of ability determinants. Journal of Applied Psychology, 77, 598-614.

Black, A. A. (2005). Spatial ability and earth science conceptual understanding. Journal of Geoscience Education, 53, 402-414.

Bodner, G. M., \& McMillen, T. L. B. (1986). Cognitive restructuring as an early stage in problem solving. Journal of Research in Science Teaching, 23, 727-737.

Bower, G. H., \& Morrow, D. G. (1990). Mental models in narrative comprehension. Science, 247, 44-48.

Carroll, J. B. (1993). A theory of cognitive abilities: The three-stratum theory. In J. B. Carroll (Ed.), Human cognitive abilities: A survey of factor-analytic studies (pp. 631-655). Cambridge: Cambridge University Press.

Carter, C. S., LaRussa, M. A., \& Bodner, G. M. (1987). A study of two measures of spatial ability as predictors of success in different levels of general chemistry. Journal of Research in Science Teaching, 24, 645-657.

Cherney, I. D. (2008). Mom, let me play more computer games: They improve my mental rotation skills. Sex Roles, 59, 776-786.

Conway, A. R. A., Kane, M. J., Bunting, M. F., Hambrick, D. Z., Wilhelm, O., \& Engle, R. W. (2005). Working memory span tasks: A methodological review and user's guide. Psychonomic Bulletin \& Review, 12, 769-786.

Cooper, L. A. (1975). Mental transformations of random twodimensional shapes. Cognitive Psychology, 7, 20-43.

Cooper, L. A., \& Shepard, R. N. (1973). Chronometric studies of the rotation of mental images. In W. G. Chase (Ed.), Visual information processing (pp. 75-176). New York: Academic Press.

Dye, M. W. G., Green, C. S., \& Bavelier, D. (2009). Increasing speed of processing with action video games. Current Directions in Psychological Science, 18, 321-326.

Electronic Arts. (2011). Word Whomp.

Feng, J., Spence, I., \& Pratt, J. (2007). Playing an action video game reduces gender differences in spatial cognition. Psychological Science, 18, 850-855.

Fery, Y. A., \& Ponserre, S. (2001). Enhancing the control of force in putting by video game training. Ergonomics, 44, 1025-1037.

Fincher-Kiefer, R. (2001). Perceptual components of situation models. Memory \& Cognition, 29, 336-343.
Fincher-Kiefer, R., \& D'Agostino, P. R. (2004). The role of visuospatial resources in generating predictive and bridging inferences. Discourse Processes, 37, 205-224.

French, J. W., Ekstrom, R. B., \& Price, L. A. (1963). Kit of reference tests for cognitive factors. Princeton: Educational Testing Service.

Friedman, L. (1995). The space factor in mathematics: Gender differences. Review of Educational Research, 65, 22-50.

Friedman, N. P., \& Miyake, A. (2000). Differential roles for spatial and verbal working memory in the comprehension of spatial descriptions. Journal of Experimental Psychology: General, 129, 61-83.

Gentner, D., \& Stevens, A. L. (Eds.). (1983). Mental models. Hillsdale: Erlbaum.

Ginn, S. R., \& Pickens, S. J. (2005). Relationships between spatial activities and scores on the mental rotation test as a function of sex. Perceptual and Motor Skills, 100, 877-881.

Gopher, D., Weil, M., \& Bareket, T. (1994). Transfer of skill from a computer game trainer to flight. Human Factors, 36, 387-405.

Green, C. S., \& Bavelier, D. (2003). Action video games modify visual selective attention. Nature, 423, 534-537.

Haenggi, D., Kintsch, W., \& Gernsbacher, M. A. (1995). Spatial situation models and text comprehension. Discourse Processes, 19, 173-199.

Hegarty, M. (1992). Mental animation: Inferring motion from static diagrams of mechanical systems. Journal of Experimental Psychology: Learning, Memory, and Cognition, 18, 1084-1102.

Hegarty, M., Keehner, M., Khooshabeh, P., \& Montello, D. R. (2009). How spatial abilities enhance, and are enhanced by, dental education. Learning and Individual Differences, 19, 61-70.

Hegarty, M., \& Sims, V. K. (1994). Individual differences in mental animation during mechanical reasoning. Memory \& Cognition, 22, 411-430.

Hegarty, M., \& Steinhoff, K. (1997). Individual differences in use of diagrams as memory in mechanical reasoning. Learning and Individual Differences, 9, 19-44.

Hegarty, M., \& Waller, D. (2006). Individual differences in spatial abilities. In P. Shah \& A. Miyake (Eds.), Handbook of visuospatial thinking (pp. 121-169). New York: Cambridge University Press.

Just, M. A., \& Carpenter, P. A. (1985). Cognitive coordinate systems: Accounts of mental rotation and individual differences in spatial ability. Psychological Review, 92, 137-172.

Kozhevnikov, M., \& Hegarty, M. (2001). A dissociation between object manipulation spatial ability and spatial orientation ability. Memory \& Cognition, 29, 745-756.

Kozhevnikov, M., Motes, M. A., \& Hegarty, M. (2007). Spatial visualization in physics problem solving. Cognitive Science, 31, 549-579.

Kozma, R., \& Russell, J. (2005). Multimedia learning of chemistry. In R. Mayer (Ed.), Cambridge handbook of multimedia learning (pp. 409-428). New York: Cambridge University Press.

Mathewson, J. H. (1999). Visual-spatial thinking: An aspect of science education overlooked by educators. Science Education, 83, 33-54.

Microsoft. (2001). Halo: Combat Evolved.

Miyake, A., Friedman, N. P., Rettinger, D. A., Shah, P., \& Hegarty, M. (2001). How are visuospatial working memory, executive functioning, and spatial abilities related? A latent-variable analysis. Journal of Experimental Psychology: General, 130, 621-640.

Mumaw, R. J., Pellegrino, J. W., Kail, R. V., \& Carter, P. (1984). Different slopes for different folks: Process analysis of spatial aptitude. Memory \& Cognition, 12, 515-521.

Okagaki, L., \& Frensch, P. A. (1994). Effects of video game playing on measures of spatial performance: Gender effects in late adolescence. Journal of Applied Developmental Psychology, 15, 33-58. 
Pellegrino, J. W., \& Hunt, E. B. (1991). Cognitive models for understanding and assessing spatial abilities. In H. A. H. Rowe (Ed.), Intelligence: Reconceptualization and measurement (pp. 203-225). Mahwah: Erlbaum.

Pribyl, J. R., \& Bodner, G. M. (1987). Spatial ability and its role in organic chemistry: A study of four organic courses. Journal of Research in Science Teaching, 24, 229-240.

Rosenberg, B. H., Landsittel, D. S., \& Averch, T. D. (2005). Can video games be used to predict or improve laparoscopic skills? Journal of Endourology, 19, 372-376.

Rosser, J. C., Lynch, P. J., Cuddihy, L., Gentile, D. A., Klonsky, J., \& Merrell, R. (2007). The impact of video games on training surgeons in the 21st century. Archives of Surgery, 142, 181-186.

Sanchez, C. A., \& Branaghan, R. J. (2009). The interaction of map resolution and spatial abilities on map learning. International Journal of Human Computer Studies, 67, 475-481.

Sanchez, C. A., \& Wiley, J. (2010). Sex differences in science learning: Closing the gap through animations. Learning and Individual Differences, 20, 271-275.

Sibley, D. F. (2005). Visual abilities and misconceptions about plate tectonics. Journal of Geoscience Education, 53, 471-477.

Stumpf, H., \& Eliot, J. (1995). Gender-related differences in spatial ability and $k$ factor of general spatial ability in a population of academically talented students. Personality and Individual Differences, 19, 33-45.
Terlecki, M. S., Newcombe, N. S., \& Little, M. (2008). Durable and generalized effects of spatial experience on mental rotation: Gender differences in growth patterns. Applied Cognitive Psychology, 22, 996-1013.

Tversky, B. (1991). Spatial mental models. In G. H. Bower (Ed.), The psychology of learning and motivation: Advances in research and theory (pp. 109-145). New York: Academic Press.

Unsworth, N., Heitz, R. P., Schrock, J. C., \& Engle, R. W. (2005). An automated version of the operation span task. Behavior Research Methods, 37, 498-505.

Wiley, J. (2001). Supporting understanding through task and browser design. In Proceedings of the 23rd Annual Conference of the Cognitive Science Society (pp. 1136-1143). Hillsdale: Erlbaum.

Wiley, J., Goldman, S. R., Graesser, A. C., Sanchez, C. A., Ash, I. K., \& Hemmerich, J. (2009). Source evaluation, comprehension, and learning in internet science inquiry tasks. American Educational Research Journal, 46, 1060-1106.

Wright, R., Thompson, W. L., Ganis, G., Newcombe, N. S., \& Kosslyn, S. M. (2008). Training generalized spatial skills. Psychonomic Bulletin \& Review, 15, 763-771.

Wu, H., \& Shah, P. (2004). Exploring visuospatial thinking in chemistry learning. Science Education, 88, 465-492.

Zacks, J. M., Mires, J., Tversky, B., \& Hazeltine, E. (2002). Mental spatial transformations of objects and perspective. Spatial Cognition and Computation, 2, 315-322. 